\section{Superfluidity of Grain Boundaries and Supersolid Behavior}

\author{
S. Sasaki, ${ }^{1 *}$ R. Ishiguro, ${ }^{1}$ † F. Caupin, ${ }^{1}$ H. J. Maris, ${ }^{1,2}$ S. Balibar ${ }^{1 *}$
}

When two communicating vessels are filled to a different height with liquid, the two levels equilibrate because the liquid can flow. We have looked for such equilibration with solid ${ }^{4} \mathrm{He}$. For crystals with no grain boundaries, we see no flow of mass, whereas for crystals containing several grain boundaries, we detect a mass flow. Our results suggest that the transport of mass is due to the superfluidity of grain boundaries.

$\mathrm{T}$ he principle of communicating vessels states that if a vertical open tube is half-immersed in a liquid bath, the liquid levels inside and outside the tube equilibrate to a common height. If the top of the tube is closed, the pressure $(P)$ above the liquid can be different inside and outside the tube, causing the levels to stay at different heights. This observation was used by Evangelista Torricelli to devise the first barometer in 1643 . Furthermore, if all of the air surrounding the setup is removed, then the liquid is in equilibrium with its vapor at a well-defined $P$ (the saturated vapor pressure), and the difference in height $(h)$ vanishes. We have performed a similar experiment with ${ }^{4} \mathrm{He}$ at its liquid/solid equilibrium. We found that hydrostatic equilibrium can be achieved via frictionless mass flow through the solid. These results provide further evidence for the "supersolid" behavior reported by $\mathrm{Kim}$ and Chan $(\mathrm{KC})(1,2)$ and now confirmed by three other experimental groups (3-5). However, we have shown that mass transport probably takes place within superfluid grain boundaries (GBs) ( 6 ) and is quite different from the scenario of BoseEinstein condensation of vacancies proposed by others (7-10).

$\mathrm{KC}$ studied a torsional oscillator filled with solid ${ }^{4} \mathrm{He}$ and determined that $1 \%$ of their sample mass was superfluid below $50 \mathrm{mK}$ because it decoupled from the oscillations of its container $(1,2,11)$. They called this phenomenon supersolidity, but its interpretation remains controversial $(3,6,12-16)$. In particular, other work (3) has found that supersolid behavior was no longer observed when their crystals were annealed for 10 hours between 1.4 and $1.5 \mathrm{~K}$. In (3), it was concluded that crystal defects are

${ }^{1}$ Laboratoire de Physique Statistique de I'Ecole Normale Supérieure, Associé aux Universités Paris 6 et Paris 7 et au CNRS, 24 Rue Lhomond, 75231 Paris Cedex 05, France. ${ }^{2}$ Department of Physics, Brown University, Providence, RI 02912, USA.

*To whom correspondence should be addressed. E-mail: ssasaki@lps.ens.fr (S.S.); balibar@lps.ens.fr (S.B.)

†Present address: Graduate School of Science, Osaka City University, Osaka 558-8585, Japan. essential for the existence of supersolidity, although $\mathrm{KC}$ did not observe any effect of annealing. After KC's observations, another study looked for mass flow inside porous Vycor glass filled with solid ${ }^{4} \mathrm{He}$ (17) but found no mass flow at low temperature. This experiment was perhaps sensitive to the pinning of the lattice to the side walls. Although lattice pinning was probably not as important in a follow-up experiment in which the geometry of the setup was more open (18), we decided to look for mass flow in a large tube and in a situation where no lattice deformation was necessary.

In our experiment, we partially filled a glass test tube (inner diameter $D=1 \mathrm{~cm}$ ) with solid ${ }^{4} \mathrm{He}$ grown from liquid ${ }^{4} \mathrm{He}$ (Fig. 1) and observed it with a charge-coupled device camera through the windows of a cryostat (Fig. 2). We started with a configuration where the liquid/ solid interface inside the tube was higher than outside. If mass can flow through the solid, gravity drives the system to the equilibrium state where both interfaces are at the same

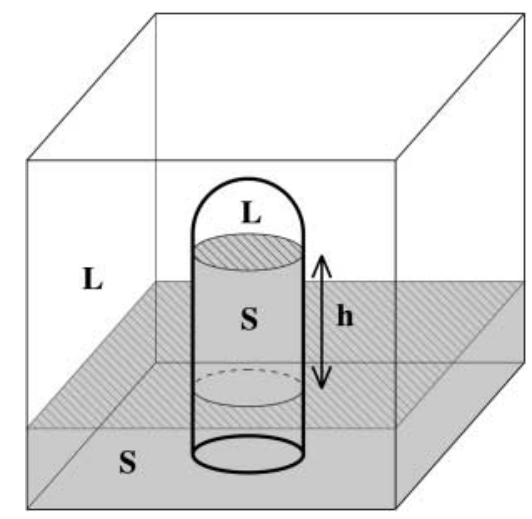

Fig. 1. A schematic of a 1-cm-diameter test tube partly filled with solid helium (S) in contact with liquid helium (L) above. After preparing the liquid/solid interface (hatched area) at a higher level inside the tube than outside (distance represented by $h$ ), we observed a relaxation to the same level on both sides of the tube if a continuous path along GBs connected these two regions. level. This is because thermal effects are negligible (19-21). Furthermore, the crystal cannot slip against the wall tube because it is blocked by the geometry of the setup $(22,23)$. The levels can move only by local crystallization and melting, with the lattice being immobile. However, because the solid density $\left(\rho_{\mathrm{C}}\right)$ is $10 \%$ higher than the liquid density $\left(\rho_{L}\right)(24)$, relaxation requires mass flow from the liquid inside the tube to the liquid outside. If the top of the tube were open, mass transport through the bulk liquid would be very efficient and, because the liquid/solid interface is very mobile at low temperature, the levels would relax with a very short time constant $(0.2 \mu$ s at $50 \mathrm{mK})(25)$. But the top of the tube is closed, and the relaxation is limited by the resistance to mass flow through the solid. As we shall see, there is no substantial mass flow along the glass/helium interface in our experiment.

Although a similar experiment provided a negative result (26), the crystal relaxation in that experiment required crystal growth into a narrow space. During growth, crystals are usually faceted, and facets are easily pinned by surface defects (25). In our case, the crystal has to melt inside the tube, so that facets disappear.

To prepare solid ${ }^{4} \mathrm{He}$ inside the tube, the pressure outside the tube needs to be much higher than inside. To satisfy this condition, for 4 to $10 \mathrm{~s}$ the melting pressure $\left(P_{\mathrm{m}}\right)$ was 25.7 bars at $1.3 \mathrm{~K}$ inside the tube and 26 bars at $1.4 \mathrm{~K}$ outside the tube (24). This could be achieved during growth by temporarily increasing the flow rate into the cell through its fill line. This procedure was repeated several times in order to solidify most of the helium inside the tube. Each time, the crystal grew at a speed in the

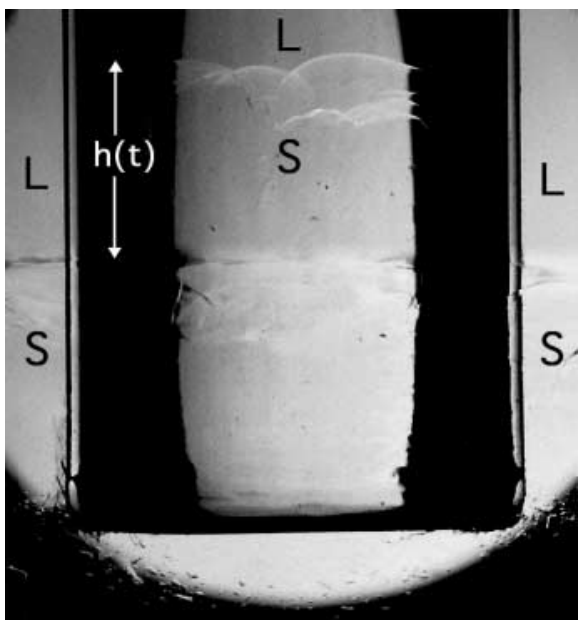

Fig. 2. Photograph of solid helium prepared at $1.3 \mathrm{~K}$. The interface between the liquid $(\mathrm{L})$ and the solid (S) shows cusps where GBs emerge. Outside the tube, which appears black because of refraction effects, the interface is lower than inside by $h(t)$. Seen on either side and in front, the interface completely surrounds the tube. 
range from 0.1 to $1 \mathrm{~mm} / \mathrm{s}$ and several GBs formed, as shown by the presence of cusps at the liquid/solid interface. GBs have a surface tension comparable to that of the liquid/solid interface, so mechanical equilibrium induces macroscopic cusps where GBs meet the liquid/ solid interface (Fig. 2). We often observed that cusps disappeared spontaneously, with a typical time constant of 1 hour. It seems that some GBs were able to move transversely before vanishing either on side walls or at the liquid/ solid interface, whereas other GBs were pinned, probably to walls, and stayed immobile. The GB motion can be driven by residual stresses in the polycrystalline samples.

After using the above method to prepare crystals, closing the fill line, cooling to $50 \mathrm{mK}$, melting the outside part of the solid by opening the fill line, and finally bringing its level to $1 \mathrm{~cm}$ below the level inside, we watched for a possible relaxation of the interface levels.

We studied 13 crystals, 10 of which showed no or very few cusps inside the tube 2 hours after preparation. For these crystals of good quality, we observed no relaxation. The $1-\mathrm{cm}$ $h(t)$ stayed constant within $50 \mu \mathrm{m}$ over a time $(t)$ of 4 hours; the interface velocity $(V)$ was less than $3.5 \times 10^{-3} \mu \mathrm{m} \mathrm{s}^{-1}$. This velocity is at least 300 times less than that expected from KC's picture, where the supersolid density $\left(\rho_{\mathrm{s}}\right)$ is $1 \%$ and the supersolid mass flows through the bulk crystal at a critical velocity $v_{\mathrm{c}}=10 \mu \mathrm{m} \mathrm{s}^{-1}$. In a tube of cross section $S=\pi D^{2} / 4$, this would give a mass current $J=S \rho_{\mathrm{s}} v_{\mathrm{c}} . J$ and $V$ are related through $J=S\left(\rho_{\mathrm{C}}-\rho_{\mathrm{L}}\right) V$, so that the corresponding interface velocity would be $V=$ $\rho_{\mathrm{s}} v_{\mathrm{c}} /\left(\rho_{\mathrm{C}}-\rho_{\mathrm{L}}\right)=1 \mu \mathrm{m} \mathrm{s}^{-1}$. Our results are not consistent with simple interpretations of KC's experiment in terms of a $1 \%$ equilibrium density of vacancies forming a Bose-Einstein condensate in single crystals (10).

Fig. 3. The $h(t)$ between the liquid/solid interface inside and outside the tube as a function of time for two different crystals. Error bars indicate the accuracy at which heights are measured $(+/-50$ $\mu \mathrm{m})$; for crystal 2, they are smaller than the symbol size.
For three crystals of medium quality, we observed a definite mass transport through the solid. The relaxation of two crystals studied at $50 \mathrm{mK}$ is shown (Fig. 3, crystals 1 and 2). Inside the tube, crystal 1 showed one cusp, which vanished after $1000 \mathrm{~s}$. As long as this cusp was visible, the $h(t)$ between the interface level inside and outside the tube relaxed at a constant velocity $(0.6 \mu \mathrm{m} / \mathrm{s})$ (Fig. 3). When the cusp disappeared, the relaxation stopped. Crystal 2 showed many cusps and relaxed much faster than crystal 1 . The interface moved first at 6 $\mu \mathrm{m} / \mathrm{s}$ and reached an even more disordered region of the crystal after $500 \mathrm{~s}$, where more cusps were visible and the velocity changed to $11 \mu \mathrm{m} / \mathrm{s}$. Finally, $h(t)$ reached 0 and stopped. Because a viscous fluid would relax exponentially, a relaxation at constant velocity, on the contrary, is characteristic of superfluid flow at its critical velocity. At the end of the relaxation, several cusps were still visible inside the tube. The equilibrium position at $h=0$ confirmed that the temperature difference between the two sides of the tube was negligible (19). We also tried to regrow the crystal inside by slowly injecting more ${ }^{4} \mathrm{He}$ through the fill line at a constant temperature, but facets developed, which blocked the growth inside.

If the interface with the glass wall is the same as for crystals of good quality, the relaxation in these two crystals should be due to superfluid flow along a thin continuous path that follows GBs across the whole solid. For crystal 1 , a single path was cut after a partial relaxation. The mass flow is limited by $v_{\mathrm{c}}$

${ }_{\mathrm{c}}^{v_{\mathrm{GB}}}=\frac{\pi D^{2}}{4 e w} \frac{\rho_{\mathrm{C}}-\rho_{\mathrm{L}}}{\rho_{\mathrm{s}}} V=1.5 \frac{a}{e} \frac{D}{w} \frac{\rho_{\mathrm{C}}}{\rho_{\mathrm{s}}} \mathrm{m} / \mathrm{s}$

inside a GB that has a width $w$, a thickness $e$, and a superfluid density $\rho_{\mathrm{s}}$; $a$ is equal to $3 \AA$, which is the thickness of one atomic layer. The above result is consistent with the study of liquid films of atomic thickness adsorbed on a wall (27). Although these films had a free surface, similar critical velocities were found (up to $2 \mathrm{~m} / \mathrm{s}$ ).

Having found evidence for a direct current of mass through solid ${ }^{4} \mathrm{He}$ samples at $50 \mathrm{mK}$, the temperature at which $\mathrm{KC}$ first observed supersolidity, we looked for a possible temperature dependence. It is difficult to keep a single GB fixed and to study its properties as a function of temperature, but we have observed similar superflow at $1.13 \mathrm{~K}$ in a third crystal that had many defects. This crystal changed relaxation speed several times, and its $h(t)$ finally relaxed to zero at $0.9 \mu \mathrm{m} / \mathrm{s}$ after $4500 \mathrm{~s}$. This observation suggests that GBs are thick enough at $P_{\mathrm{m}}$ for their superfluid transition temperature $\left(T_{\mathrm{c}}\right)$ to be close to that of bulk liquid ${ }^{4} \mathrm{He}$. However, the properties of the GBs might depend on the misorientation between the adjacent crystals.

Let us now consider the possibility that there is a superfluid film between the glass wall and solid helium $(14,15)$. Because solid ${ }^{4} \mathrm{He}$ partially wets walls (25), a superfluid film at the glass/helium interface necessarily has a microscopic thickness $e$ on the order of $a$. Because crystals of good quality do not relax, we found an upper bound for $v_{\mathrm{c}}^{\mathrm{W}}$ near the glass wall

$$
v_{\mathrm{c}}^{\mathrm{W}}=\frac{D}{4 e} \frac{\rho_{\mathrm{C}}-\rho_{\mathrm{L}}}{\rho_{\mathrm{s}}} V<0.28 \frac{a}{e} \frac{\rho_{\mathrm{C}}}{\rho_{\mathrm{s}}} \mathrm{cm} / \mathrm{s}
$$

If $e$ were equal to 4 to $8 a$ as proposed in (14), the superfluid film near the wall should be similar to the free liquid films in which the $v_{\mathrm{c}}$ is much larger (27). On the contrary, if we assume $e=a$ and $\rho_{\mathrm{s}} / \rho_{\mathrm{C}} \approx 0.04$ as proposed in (15), we find $v_{\mathrm{c}}^{\mathrm{W}}<7 \mathrm{~cm} / \mathrm{s}$. This is possible if the superfluid film near the glass wall is much thinner and denser than inside a GB (28) but, in this case, it is probably not thick enough to explain KC's results. It therefore appears difficult to explain the results of both $\mathrm{KC}$ 's experiments and ours in terms of a superfluid film at the glass/helium interface.

Our results suggest that GBs are superfluid, so that ${ }^{4} \mathrm{He}$ crystals of medium quality are supersolid at the liquid/solid equilibrium pressure $P_{\mathrm{m}}$; that is, mass transport through them without dissipation is possible. But they are not "supercrystalline" because transport does not occur through their crystalline part. However, the connection of our results with other observations of supersolidity $(1-5)$ is not straightforward, because our measurements were taken at $P_{\mathrm{m}}$, whereas others were taken at higher pressures. We now need to study the superfluidity inside GBs more carefully. We expect $e$ and $v_{\mathrm{c}}^{\mathrm{GB}}$ to have a sharp variation near $P_{\mathrm{m}}$, 
where these quantities should be larger than at $P>P_{\mathrm{m}}$, away from the stability region of the liquid. It is also possible that the superfluid transition temperature $T_{\mathrm{c}}^{\mathrm{GB}}$ is considerably lower than $1 \mathrm{~K}$ at high $P$, where superfluidity may concern only a fraction of a monolayer. Unfortunately, to work with a single GB while varying the temperature and $P$ is not easy. One should also study the effect of ${ }^{3} \mathrm{He}$ impurities because these should adsorb on GBs and modify their superfluid properties.

Let us finally consider the values of $\rho_{\mathrm{s}}$ and $v_{\mathrm{c}}$ found in torsional oscillator experiments (1-5). If the superflow occurs along the GBs in these experiments as well, $v_{\mathrm{c}}$ needs to decrease sharply from meters per second at $P_{\mathrm{m}}$ to micrometers per second above $P_{\mathrm{m}}$. This is possible if the thickness of GBs also decreases as a function of $P$. A $1 \%$ superfluid density can be achieved with one GB every $100 e$. We have found that, when growing crystals above $1.8 \mathrm{~K}$ from normal liquid helium, as is usually the case when using the blocked capillary method of $\mathrm{KC}$ and others, the growth is dendritic and the solid sample is snowball-like, in which the density of defects is so large that it strongly scatters light [see photograph in (5)]. It is also possible that $\mathrm{KC}$ 's experiment is insensitive to annealing because GBs are more strongly pinned in their cell. If GBs are close to each other, elastic interactions might couple them and mimic phase coherence. As a final note, we mention that $\mathrm{KC}$ observed that $\rho_{\mathrm{s}}(P)$ increases up to 55 bars before decreasing at higher $P$. The increase could be due to an increasing number of GBs and the decrease to the vanishing of superfluidity around 200 bars (29).

\section{References and Notes}

1. E. Kim, M. H. W. Chan, Nature 427, 225 (2004).

2. E. Kim, M. H. W. Chan, Science 305, 1941 (2004).

3. A. S. C. Rittner, ]. D. Reppy, published online 22 April 2006 (http://arxiv.org/PS_cache/cond-mat/pdf/0604/ 0604528.pdf).

4. K. Shirahama, M. Kondo, S. Takada, Y. Shibayama, American Physical Society, Baltimore, MD, 14 March 2006 (abstract G41.00007).

5. A. Cho, Science 311, 1693 (2006)

6. E. Burovski, E. Kozik, A. Kuklov, N. Prokof'ev, B. Svistunov, Phys. Rev. Lett. 94, 165301 (2005).

7. A. F. Andreev, I. M. Lifshitz, Sov. Phys. JETP 29, 1107 (1969).

8. G. V. Chester, Phys. Rev. A. 2, 256 (1970).

9. A. J. Leggett, Phys. Rev. Lett. 25, 1543 (1970).

10. D. E. Galli, L. Reatto, Phys. Rev. Lett. 96, 165301 (2006).

11. This is when $\mathrm{KC}$ used natural ${ }^{4} \mathrm{He}$ containing $\approx 0.1$ parts per million of ${ }^{3} \mathrm{He}$ impurities. We used the same natural ${ }^{4} \mathrm{He}$.

12. B. K. Clark, D. M. Ceperley, Phys. Rev. Lett. 96, 105302 (2006).

13. M. Boninsegni, N. Prokof'ev, B. Svistunov, Phys. Rev. Lett. 96, 105301 (2006).

14. G. Dash, ]. S. Wettlaufer, Phys. Rev. Lett. 94, 235301 (2005).

15. S. A. Khairallah, D. M. Ceperley, Phys. Rev. Lett. 95, 185301 (2005).

16. P. W. Anderson, W. F. Brinkman, D. A. Huse, Science 310, 1164 (2005).

17. ]. Day, T. Herman, ]. Beamish, Phys. Rev. Lett. 95 035301 (2005).

18. ]. Day, ]. Beamish, Phys. Rev. Lett. 96, 105304 (2006)

19. Because of radiation through the windows, there is a small heat input inside the tube, but it is less than $1 \mu \mathrm{W}$. In principle, $P_{\mathrm{m}}$ inside the tube could thus be different from that outside and maintain an $h(t)$ between the two levels. However, given the heat conductivity of the solid (20) and the melting curve $P_{m}(T)(21)$, where $T$ is temperature, one finds that the level difference is less than $3 \mu \mathrm{m}$ below $0.5 \mathrm{~K}$. We thus expect thermal effects to be unimportant and gravity to be the only relevant parameter governing the shape evolution.

20. B. Bertman, H. A. Fairbank, C. W. White, M. J. Crooks, Phys. Rev. 142, 74 (1966).

21. H. N. Hanson, ]. E. Berthold, G. M. Seidel, H. J. Maris, Phys. Rev. B 14, 1911 (1976).

22. In liquid ${ }^{4} \mathrm{He}$, a $1-\mathrm{cm}$ difference in height induces a difference in $P$ of $2 \times 10^{-4}$ bars, which is very small compared to the Young modulus $E=300$ bars of ${ }^{4} \mathrm{He}$ crystals (23). As a consequence, it cannot induce the large plastic deformation that would be necessary for the crystal to flow around the tube edge.

23. H. J. Maris, T. E. Huber, J. Low Temp. Phys. 48, 99 (1982).

24. E. R. Grilly, J. Low Temp. Phys. 11, 33 (1973).

25. S. Balibar, H. Alles, A. Y. Parshin, Rev. Mod. Phys. 77, 317 (2005)

26. G. Bonfait, H. Godfrin, B. Castaing, J. Phys. (Paris) 50 1997 (1989).

27. K. Telschow, I. Rudnick, T. G. Wang, Phys. Rev. Lett. 32, 1292 (1974)

28. K. A. Gillis, S. M. Volz, J. M. Mochel, Phys. Rev. B 40, 6684 (1989).

29. R. Ishiguro, F. Caupin, S. Balibar, Europhys. Lett. 75, 91 (2006)

30. We acknowledge support from Agence Nationale de la Recherche grant 05-BLAN-0084-01 and NSF grant DMR-0305115. R.I. acknowledges support from the Japan Society for the Promotion of Science for a Postdoctoral Fellowship for Research Abroad (April 2004 to March 2006)

5 June 2006; accepted 19 July 2006

Published online 27 July 2006

$10.1126 /$ science. 1130879

Include this information when citing this paper.

\section{Detection, Stimulation, and Inhibition of Neuronal Signals with High-Density Nanowire Transistor Arrays}

\author{
Fernando Patolsky, ${ }^{1 *}$ Brian P. Timko, ${ }^{1 *}$ Guihua Yu, ${ }^{1}$ Ying Fang, ${ }^{1}$ Andrew B. Greytak, ${ }^{1}$ \\ Gengfeng Zheng, ${ }^{1}$ Charles M. Lieber ${ }^{1,2} \dagger$
}

We report electrical properties of hybrid structures consisting of arrays of nanowire field-effect transistors integrated with the individual axons and dendrites of live mammalian neurons, where each nanoscale junction can be used for spatially resolved, highly sensitive detection, stimulation, and/or inhibition of neuronal signal propagation. Arrays of nanowire-neuron junctions enable simultaneous measurement of the rate, amplitude, and shape of signals propagating along individual axons and dendrites. The configuration of nanowire-axon junctions in arrays, as both inputs and outputs, makes possible controlled studies of partial to complete inhibition of signal propagation by both local electrical and chemical stimuli. In addition, nanowire-axon junction arrays were integrated and tested at a level of at least 50 "artificial synapses" per neuron.

$\mathrm{E}$ lectrophysiological measurements made with micropipette electrodes and microfabricated electrode arrays play an important role in understanding signal propagation through individual neurons and neuronal networks (1-5). Micropipette electrodes can stimulate and record intracellular and extracellular potentials in vitro and in vivo with relatively good spatial resolution of $\sim 100 \mathrm{~nm}$ per pipette and $\geq 10 \mu \mathrm{m}$ between two pipettes $(2,6-8)$, yet they are difficult to multiplex. Microfabricated structures, such as electrode and field-effect transistor (FET) arrays, have potential for largescale multiplexing and have enabled recording from both individual neurons and networks (3-5, 9-14). However, these structures have rela- tively large sizes $(\sim 10 \mu \mathrm{m}$ and larger on edge), and their interelectrode spacing $(>10 \mu \mathrm{m})$ has precluded detection and stimulation of neuronal activity at the level of individual axons and/or dendrites.

For a FET array to be used to stimulate, inhibit, and record neuronal signals from numerous locations along the neuronal projections and cell body, the gate dimensions should ideally be on the nanometer scale, and appropriate contact between the neuron and the array must be made. Silicon nanowire (SiNW) FETs (15) have been used to detect chemical and biological species (even single virus particles) in solution (16-19). We show that we can pattern arrays of SiNW FETs on a substrate and passivate the arrays such that they will function in cell-culture media. Polylysine patterning allowed us to direct the growth of rat neurons to ensure that numerous SiNW FET contacts were made to the same neuron, rather than rely on fortuitous overlap. Because the contact length along an

${ }^{1}$ Department of Chemistry and Chemical Biology, ${ }^{2}$ Division of Engineering and Applied Sciences, Harvard University, Cambridge, MA 02138, USA.

*These authors contributed equally to this work. †To whom correspondence should be addressed. E-mail: cml@cmliris.harvard.edu 